\title{
Cooperative recombination of electron-hole pairs in semiconductor quantum wells under quantizing magnetic fields
}

\author{
Y. D. Jho, ${ }^{1, *}$ X. Wang, ${ }^{2}$ D. H. Reitze,,${ }^{2,3}$ J. Kono, ${ }^{4}$ A. A. Belyanin, ${ }^{5}$ V. V. Kocharovsky,,${ }^{5,6}$ Vl. V. Kocharovsky,${ }^{6}$ and \\ G. S. Solomon ${ }^{7}$ \\ ${ }^{1}$ Department of Information and Communications, Gwangju Institute of Science and Technology, Gwangju 500-712, Republic of Korea \\ ${ }^{2}$ Department of Physics, University of Florida, Gainesville, Florida 32611, USA \\ ${ }^{3}$ National High Magnetic Field Laboratory, Florida State University, Tallahassee, Florida 32310, USA \\ ${ }^{4}$ Department of Electrical and Computer Engineering, Rice University, Houston, Texas 77005, USA \\ ${ }^{5}$ Department of Physics, Texas A\&M University, College Station, Texas 77843, USA \\ ${ }^{6}$ Institute of Applied Physics, Russian Academy of Sciences, 603950 Nizhny Novgorod, Russia \\ ${ }^{7}$ Joint Quantum Institute, NIST \& University of Maryland, Gaithersburg, Maryland 20899, USA \\ (Received 28 July 2009; revised manuscript received 14 February 2010; published 19 April 2010)
}

\begin{abstract}
We present results of detailed investigations of light emission from semiconductor multiple quantum wells at low temperatures and high magnetic fields excited by intense femtosecond laser pulses. The intensity and linewidth as well as the directional and statistical properties of photoemission strongly depended on the magnetic field strength and pump laser fluence. We also investigated the effects of spot size, temperature, excitation geometry, and excitation pulse width on the emission properties. The results suggest that the initially incoherent photoexcited electron-hole pairs spontaneously form a macroscopic coherent state upon relaxation into the low-lying magnetoexcitonic states, followed by the emission of a superfluorescent burst of radiation. We have developed a theoretical model for superfluorescent emission from semiconductor quantum wells, which successfully explained the observed characteristics.
\end{abstract}

DOI: 10.1103/PhysRevB.81.155314

PACS number(s): 78.20.Ls, 78.55.-m, 78.67.-n

\section{INTRODUCTION}

Investigations of coherent phenomena in bulk and quantum-confined semiconductor systems have been made possible in the last few decades through the use of ultrafast lasers, which create and probe quantum coherent carriers on time scales faster than inherent phase-breaking (decoherence) times in these systems, typically $10-100$ fs for free carriers to a few picoseconds for excitons. During the coherent temporal regime, the photoexcited carriers retain a welldefined phase relationship with the excitation field, and the induced carrier polarization can be followed as the polarization decays, thus probing fundamental quantum decoherence and scattering. A vast array of ultrafast coherent dynamics in semiconductors has been explored. ${ }^{1}$ More specifically, investigations of coherent quantum optics phenomena in condensed matter and mesoscopic systems are actively being pursued. $^{2}$ In particular, ultrafast semiconductor analogs of quantum optical phenomena such as Rabi flopping ${ }^{3}$ and superradiance ${ }^{4}$ have been investigated.

An equally important but much less studied phenomenon in semiconductors and nanostructures is the spontaneous formation of macroscopic quantum coherence. Under specific physical conditions, an ensemble of interacting particles can spontaneously establish quantum coherence. This situation is fundamentally different from laser-driven coherence. Typically, a spontaneous appearance of coherence occurs at a critical temperature and/or density at which the particle interactions energetically favor a single $N$-body wave function. Bose-Einstein condensation, ${ }^{5}$ superconductivity, ${ }^{6}$ and superfluidity ${ }^{7}$ are typical examples. While the observation of spontaneous quantum coherence in semiconductors has long been sought after, investigations have been heretofore ham- pered by the strong electronic interactions and concomitant rapid dephasing times in these systems. Nevertheless, investigations pursuing spontaneous coherence are important because they can reveal much about the underlying fundamental quantum-mechanical interactions of $N$-particle systems.

Superfluorescence (SF) is a well-known example in quantum optics, showing spontaneously created macroscopic quantum coherence. In SF, an incoherently prepared system of inverted two-level atoms develops large-amplitude coherent oscillations of the optical polarization starting from quantum fluctuations through the self-phasing process mediated by exchange of photons. ${ }^{8-10}$ The resultant macroscopic optical polarization decays superradiantly, ${ }^{11,12}$ producing a highly directional burst of coherent radiation. Until very recently, SF have been observed in atomic gases ${ }^{13,14}$ and rarefied impurities in crystals. ${ }^{15-17}$ Observations of SF emission from high-density systems such as semiconductor electronhole plasmas have heretofore proven difficult.

We recently reported possible evidence for SF from a dense photoexcited electron-hole plasma in $\mathrm{InGaAs} / \mathrm{GaAs}$ multiple quantum wells in strong perpendicular magnetic fields. ${ }^{18}$ Using intense femtosecond laser pulses to create a dense electron-hole plasma in $\mathrm{InGaAs} / \mathrm{GaAs}$ multiple quantum wells, combined with a strong perpendicular magnetic field to laterally confine electrons and holes and increase the density of states through Landau quantization, we observed strong emission arising from interband recombination between Landau levels (LL) in the conduction and valence bands. Based on the spectral, spatial, and statistical characteristics of the emission lines, we interpreted the emissions to be SF. Our density-dependent investigations revealed a transition from a spontaneous emission (single-particle recombination) regime at low densities and magnetic fields through 
an intermediate (and deterministic) amplified spontaneous emission (ASE) regime to a stochastically emitting SF regime at the highest densities and magnetic fields. The purpose of the present paper is to present more fully our measurements and theory.

The paper is organized as follows. We briefly describe the experimental procedures and details in Sec. II. Our experimental results are given in Sec. III, where we present a comprehensive set of magneto-optical photoluminescence (PL) spectra under a variety of conditions. In Sec. IV, we present the theoretical basis for SF from quantized electron-hole plasmas, present key physical parameters, and discuss the different density- and field-dependent emission regimes. In Sec. V, we use our theoretical model to explain the experimentally observed emission spectra as a function of magnetic field, excitation fluence and duration, excitation geometry, and temperature as well as the spatial characteristics of the emission in different regimes. Finally, we summarize our findings and conclusions in Sec. VI.

\section{EXPERIMENTAL METHODS}

The experiments were performed at the National High Magnetic Field Laboratory in Tallahassee, FL, using a $31 \mathrm{~T}$ dc resistive magnet. $\mathrm{In}_{0.2} \mathrm{Ga}_{0.8} \mathrm{As} / \mathrm{GaAs}$ multiple quantumwell (MQW) samples, consisting of 15 layers of 8-nm-thick QWs separated by $15-\mathrm{nm}$ GaAs barriers grown by molecular-beam epitaxy, were used. The magnetic fielddependent low-power CW PL and absorption spectra of these samples are thoroughly described in Refs. 19 and 20. We used a 775-nm Ti:Sapphire-based regenerative amplifier system with a repetition rate of $1 \mathrm{kHz}$ to excite the sample. The excitation wavelength was chosen such that carriers were excited into the continuum, far above the lowest quantumwell levels. Both the pulse width and fluence of the pump beam were varied. A pump fluence of $\sim 0.01 \mathrm{~mJ} / \mathrm{cm}^{2}$ is expected to generate a carrier density of $\sim 10^{12} \mathrm{~cm}^{-2}$ in our samples.

The femtosecond excitation beam was delivered through free space into the magnet and normally incident to the QWs; the QW plane was oriented perpendicular to the magnetic field. The emitted PL was collected using multimode optical fibers placed either on the opposite face (for collecting emission perpendicular to the QW plane) or on a cleaved edge of the sample (for emission in the plane of the QW) using right-angle microprisms. The collected PL was analyzed with a high-resolution grating spectrometer equipped with a charge-coupled device (CCD) detector. The collection area of the prisms was $1 \times 1 \mathrm{~mm}^{2}$ and the computed acceptance angle based on geometric considerations was $\sim 40^{\circ}$.

Most of the spectra were collected by averaging the emission from approximately 1000 pulses but single pulses (selected using a Pockels cell external to the laser) were used for excitation in experiments to probe the statistics of the directionality of the emission. The temperature of the sample was varied from 10 to $130 \mathrm{~K}$. In addition, the pump spot size as well as the spot shape (i.e., aspect ratio) were varied to examine the characteristics of the emission relative to the predicted coherence length of the electron-hole plasma and

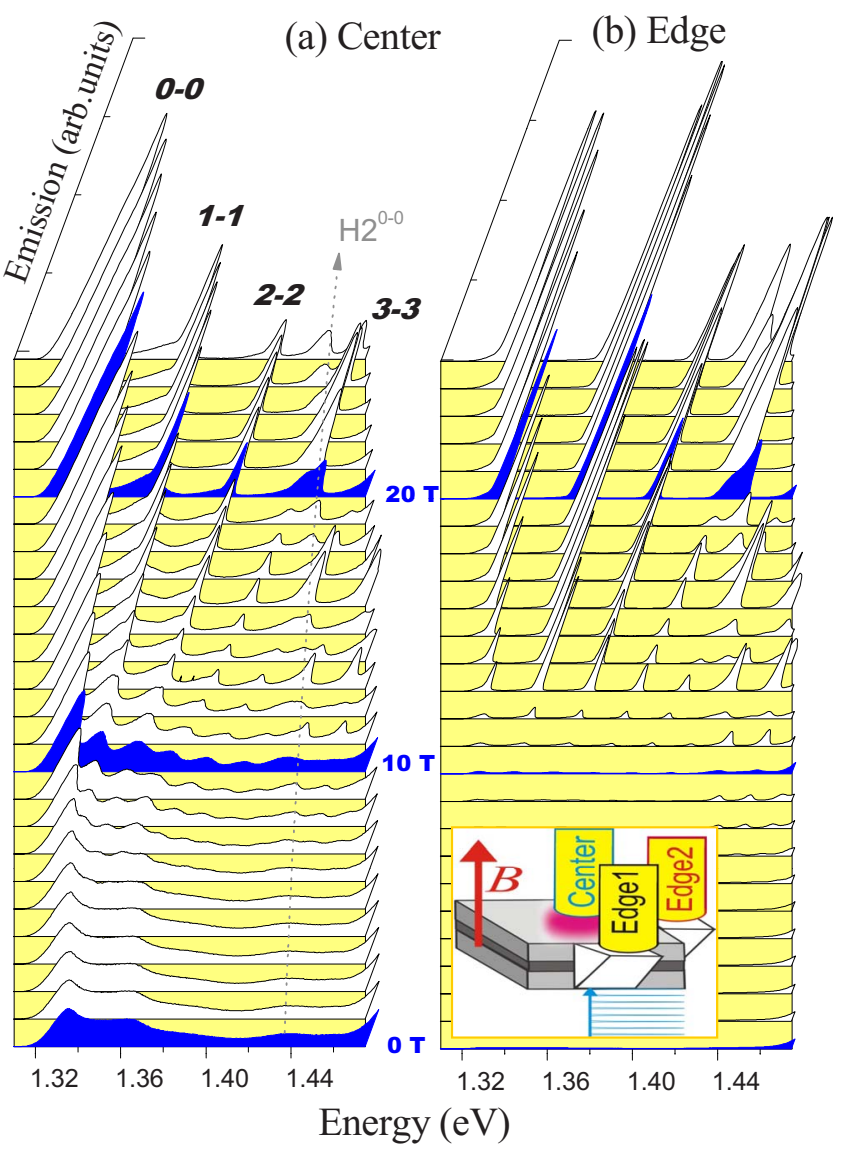

FIG. 1. (Color online) Emission spectra at various magnetic fields for a fixed pump fluence of $0.62 \mathrm{~mJ} / \mathrm{cm}^{2}$ (a) for emission through sample above the excitation spot along the laserpropagation direction and (b) for in-plane emission in an edge collection geometry. The inset of (b) shows an experimental schematic showing excitation pulses from the bottom and the collection fibers from the top. The gray dotted line denotes the position of the $0-0$ Landau level of the E2-HH2 state.

the directionality of the emitted light with respect to the sample length, respectively.

\section{EXPERIMENTAL RESULTS}

Figure 1 displays measured emission spectra at various values of magnetic field $B$ for a pump fluence of $\sim 0.62 \mathrm{~mJ} / \mathrm{cm}^{2}$ (a) from the opposite side of the sample above the pump spot (denoted by "center;" see inset) and (b) at the sample edge perpendicular to the excitation direction (edge). At low magnetic fields, well defined but broad $(\Delta E$ $\sim 9 \mathrm{meV}$ ) features are observed, similar to previous studies. ${ }^{21,22}$ However, at a threshold field of $\sim 13 \mathrm{~T}$, narrow spectral features $(\Delta E \sim 2 \mathrm{meV})$ emerge from the highenergy side of the broad peaks. The narrow features grow rapidly with increasing $B$ and become dominant at higher $B$. Although clearly visible in both the center and edge geometries, the narrow emission features are generated with much higher efficiency in the edge geometry; the integrated emission strength of the sharp feature in the $0-0 \mathrm{LL}$ at $25 \mathrm{~T}$ is 


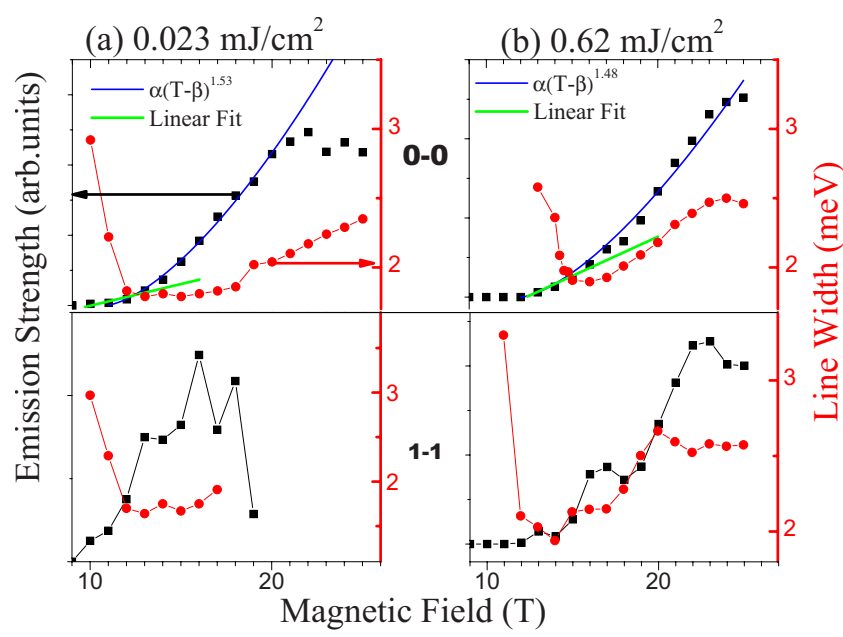

FIG. 2. (Color online) Emission strength (black squares) and linewidth (red circles) of the narrow peak from $0-0$ and 1-1 LLs versus $B$ for different pump fluence of (a) $0.24 \mathrm{~mJ} / \mathrm{cm}^{2}$ and (b) $0.62 \mathrm{~mJ} / \mathrm{cm}^{2}$. The blue and green lines represent the superlinear and linear scaling with $B$, respectively.

approximately 70 times stronger than the broad spontaneous emission.

To analyze the growth of the edge-collected signal with increasing $B$, the integrated strength from individual LLs was fitted using a Lorentzian line for the narrow, blueshifted feature and a Gaussian line for the broad, low-energy feature. A Gaussian line shape is typical for QW spontaneous emission while a Lorentzian line shape originates from homogeneously broadened, lifetime-limited lines. From the lineshape analysis, we obtained the integrated emission signal (black squares) and linewidth (red circles) traces in Fig. 2 for pump fluences of (a) $0.023 \mathrm{~mJ} / \mathrm{cm}^{2}$ and (b) $0.62 \mathrm{~mJ} / \mathrm{cm}^{2}$, respectively. At the lower fluence [Fig. 2(a)], the narrow emission 0-0 LL peak emerges at approximately $11 \mathrm{~T}$, then increases as $\propto B^{2}$ up to $\sim 20 \mathrm{~T}$. The linewidth undergoes a dramatic reduction (from 3 to $\sim 2 \mathrm{meV}$ ) as the field increases from 10 to $13 \mathrm{~T}$, followed by a slow increase as the field is further increased. (Unless noted, we will refer only to the narrow emission feature in further discussions.) The reduction of the linewidth, which is concomitant with the linear scaling of emission strength, is one of the characteristics of the ASE with increasing carrier density. The 1-1 LL feature shows similar behavior in terms of threshold magnetic field and linewidth values, although the integrated PL intensity growth cannot be definitely assigned a scaling law. Above $20 \mathrm{~T}$, the emission from the $0-0 \mathrm{LL}$ saturates and the 1-1 LL decreases dramatically, indicating that at this pump fluence cannot populate the 1-1 LL and barely fills the $0-0$ LL.

At a higher fluence of $0.62 \mathrm{~mJ} / \mathrm{cm}^{2}$ [Fig. 2(b)], the emission strength and linewidth display qualitatively similar behavior to that seen in the lower fluence case [Fig. 2(a)]. However, the 1-1 LL emission is seen to oscillate substantially as a function of field. In addition, the increase in fluence allows for the emission from $0-0$ and 1-1 LLs over the entire field range of $25 \mathrm{~T}$. Below $11 \mathrm{~T}$, narrow emission is not observed for any LL. In the case of 0-0 LL, the signal grows

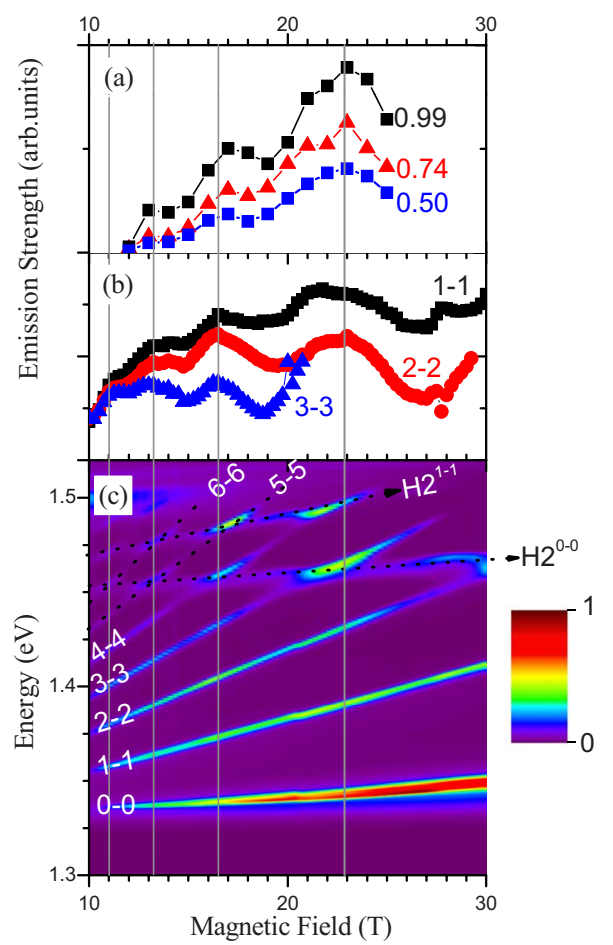

FIG. 3. (Color online) Narrow line emission strength as a function of magnetic field from 10 to $30 \mathrm{~T}$ for (a) the $1-1 \mathrm{LL}$ at various pump fluences and (b) 1-1, 2-2, and 3-3 LLs at a fixed pump fluence of $0.43 \mathrm{~mJ} / \mathrm{cm}^{2}$ obtained from a line-shape analysis of the emission data. In (c), we present a contour plot of the normalized emission strength versus field and energy for a fluence of $0.43 \mathrm{~mJ} / \mathrm{cm}^{2}$. The dashed gray lines in (c) show the mixing points between various LLs and higher sublevels and the vertical lines show the correlation between the oscillation peaks and the mixing energies.

linearly with $B$ from $12-14 \mathrm{~T}$ (green lines) and the linewidth reveals a significant correlation with the emission strength data. In the linear regime, the linewidth decreases monotonically versus $B$ until the emission becomes superlinear, where the linewidth begins to increase.

To further investigate the origin of the oscillatory emission strength behavior with field, in Fig. 3 we plot the emission strength versus magnetic field for the 1-1 LL at different fluences [Fig. 3(a)] and for different LLs at a fixed fluence [Fig. 3(b)]. Above a fluence of $0.4 \mathrm{~mJ} / \mathrm{cm}^{2}$, the oscillations are a universal feature, independent of any specific LL or excitation fluence. For comparison purposes, a contour map of the field-dependent PL emission levels is presented in Fig. 3(c). In addition to the H1 LLs (labeled 0-0, 1-1, etc.), the higher heavy-hole states $\mathrm{H} 2^{0-0}$ and $\mathrm{H} 2^{1-1}$ are observed (indicated by gray dashed lines) as reported in our previous study. ${ }^{19}$ The gray vertical lines identify the mixing energies and the oscillation peaks: a clear correlation is seen between the mixing points of the $\mathrm{H} 1-\mathrm{H} 2$ levels and the peaks in the oscillations of the emission. At points where specific $\mathrm{H} 1$ and H2 LLs cross, a significant enhancement is seen in the emission strength of those LLs.

Next we show how the strength and linewidth of the narrow emission lines depend on the excitation area. Figure 4 examines three situations, where the excitation area was var- 


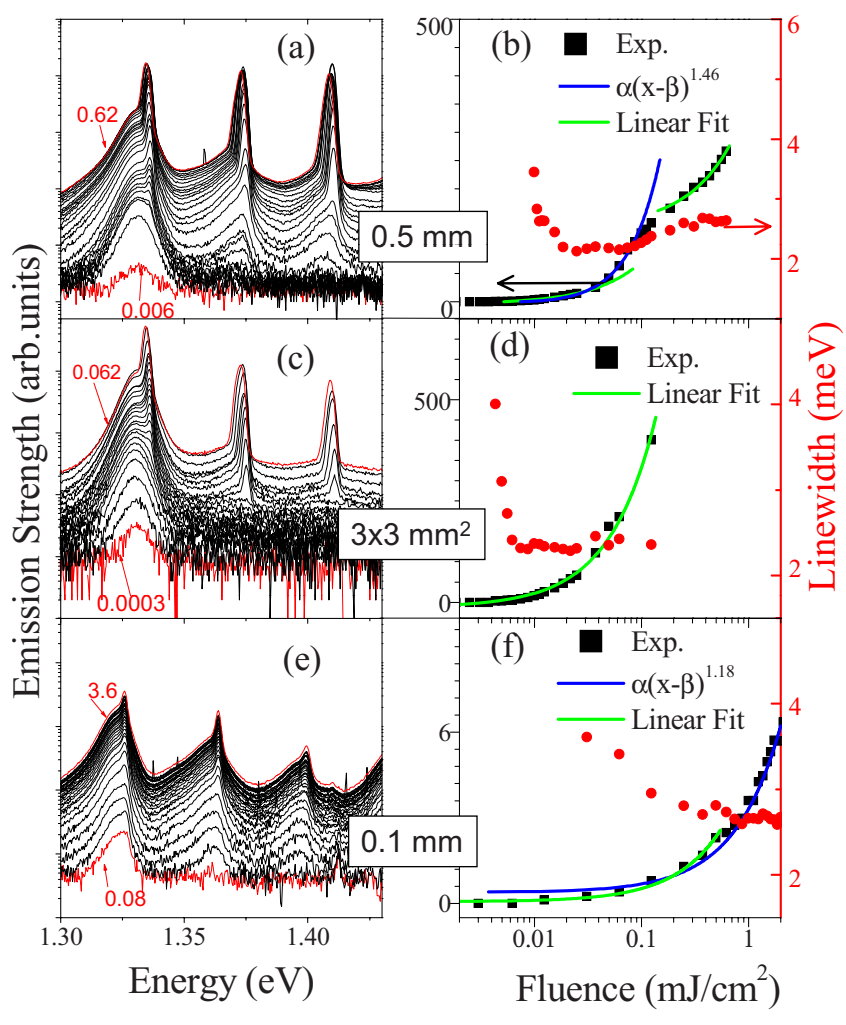

FIG. 4. (Color online) Emission strength as a function of fluence at a fixed field of $20 \mathrm{~T}$ for different spot sizes of (a) $0.5 \mathrm{~mm}$ diameter, (b) $3 \mathrm{~mm}$, and (c) $0.1 \mathrm{~mm}$ diameter. [(d)-(f)] Integrated emission intensity (black squares) and linewidth (red circles) of the narrow peak from the $0-0$ LL versus fluence for different pump spot sizes. The fluence ( $x$ axis) is plotted on a logarithmic scale. The blue (green) lines in (a)-(d) indicate the superlinear (linear) regime.

ied systematically. For these data, the magnetic field was held at $20 \mathrm{~T}$, and the fluence was varied from 0.006 to $2 \mathrm{~mJ} / \mathrm{cm}^{2}$. The left-hand column displays the obtained spectra (in log scale) while the right-hand column plots the integrated intensity and linewidth versus fluence. Qualitatively different behaviors are seen among the three cases in the fluence dependence. Figures 4(a) and 4(b) show data obtained with a pump spot size of $0.5 \mathrm{~mm}$ in diameter, showing a superlinear dependence. However, when the laser spot was increased (decreased) to $3 \mathrm{~mm}$ (0.1 $\mathrm{mm}$ diameter), both the integrated signal (black squares) and the linewidth (red circles) exhibited linear or nearly linear scaling, and in both of these cases the linewidth monotonically decreased with increasing fluence. We will show in Sec. IV C that the coherence length over which a SF pulse is formed is $\sim 0.4 \mathrm{~mm}$ and, to observe SF, it is optimal to create an inverted population using a spot of this size.

We found that the shape of the excitation region can also be used to influence emission characteristics of the narrow lines. In particular, it is possible to control the SF emission orientation through tailoring the geometry of the gain region. Using a cylindrical lens to generate a rodlike $3 \mathrm{~mm}$ $\times 0.5 \mathrm{~mm}$ excitation region, Fig. 5 shows the signal intensity as a function of angle $\theta$ from $0^{\circ}$ to $180^{\circ}$ [see the inset of Fig. 5(c)] for a fluence of $0.02 \mathrm{~mJ} / \mathrm{cm}^{2}$ and $B=25 \mathrm{~T}$. The maximum intensity follows the orientation of the long axis of
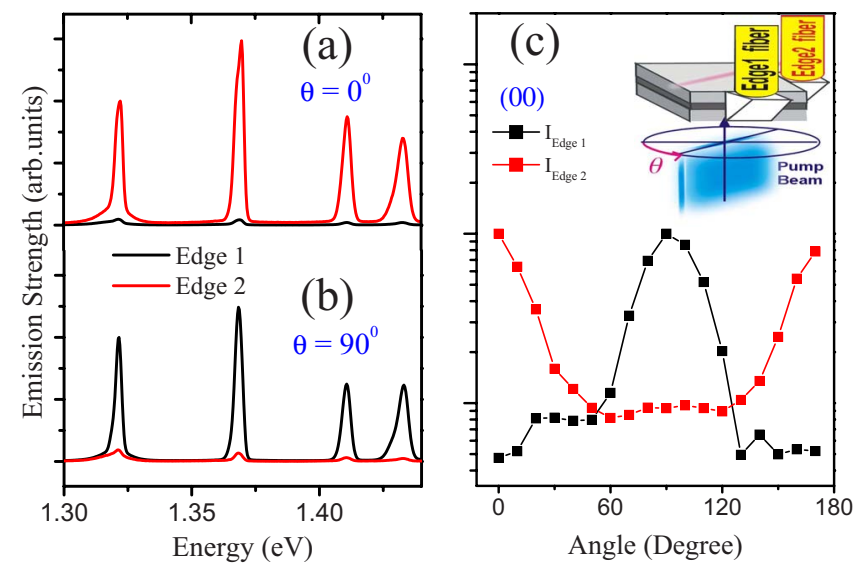

FIG. 5. (Color online) Edge emission spectra measured through two fibers placed at $\theta=(\mathrm{a}) 0^{\circ}$ and (b) $90^{\circ}$, where $\theta$ is the angle between the longer beam axis and the direction of edge 2 fiber, as shown in the inset of (c). In (c), the emission strength of $0-0$ LL is plotted for edge 1 (black) and edge 2 (red) as a function of angle.

the gain region. Tracing the output intensity displayed in Fig. 5(c) for edges 1 (black) and 2 (red), we see that the emission is highly directional with a full width at half maximum (FWHM) of $40^{\circ}$. This value, comparable to the acceptance angle of our measurement, indicates that the emission is highly directional. The variance in emitted power is $\sim 20$ between the two directions and we can conclude that the emission direction can be manipulated through tailoring of the gain geometry. However, this behavior is expected both for ASE and SF.

A more exotic feature of SF, which is not expected for ASE, is random emission directionality arising from the nondeterministic nature of spontaneous coherence. In order to verify the directionality randomness predicted for SF, we reduced the excitation pulse repetition rate down to $20 \mathrm{~Hz}$ and collected emission pulses individually on a shot-by-shot basis. The signals from the two perpendicular edges were mapped into spatially separate images in CCD detector. Figure 6(a) illustrates typical single-shot spectra of SF with a pump fluence of $9.7 \mathrm{~mJ} / \mathrm{cm}^{2}$ at $25 \mathrm{~T}$, collected through edge 1 (black) and edge 2 (red) fibers for four representative shots, chosen from 36 shots in total. In Fig. 6(b), the outputs from edge 1 and edge 2 fibers were normalized to have the same maximum although actual emission strength from edge $1 \mathrm{fi}-$ ber was weaker due to differences in the collection efficiency of each fiber.

We clearly observe that the two fiber outputs are anticorrelated over 19 different shots, which is about half of the total 36 shots. Sixteen events are those in which both edges receive significant radiation. There is only one event when both edges receive little radiation simultaneously. Over all of the shots, the maximum observed emission strength in Fig. 6(b) fluctuates as much as eight times the minimum value. This is far greater than the pump pulse fluctuation, on the order of a few percent, implying that each SF burst is very directional and randomly changing from pulse to pulse. At a lower fluence of $0.02 \mathrm{~mJ} / \mathrm{cm}^{2}$ (obtained with a $3 \times 3 \mathrm{~mm}^{2}$ spot), qualitatively different behavior is seen. Figures 6(c) and 6(d) show omnidirectional emission on every shot, as 


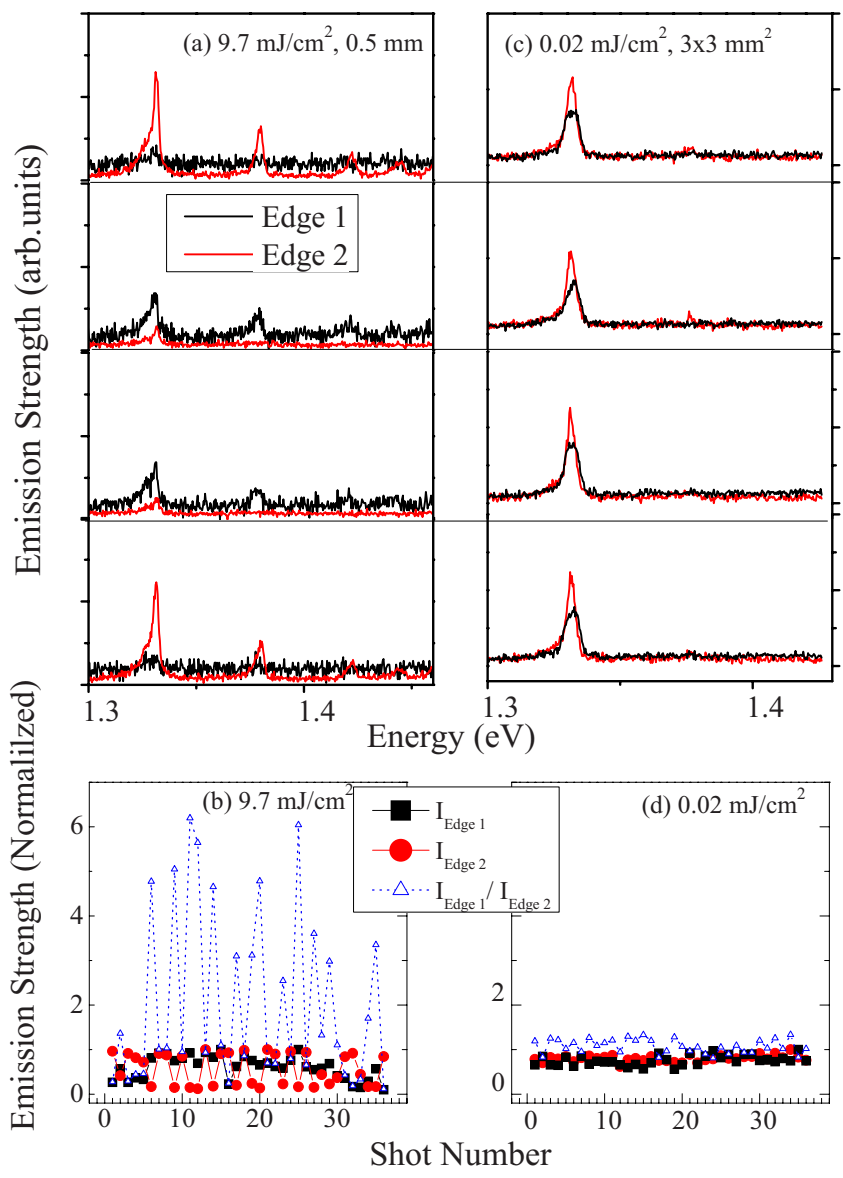

FIG. 6. (Color online) Four representative emission spectra at $25 \mathrm{~T}$ from edge 1 (black) and edge 2 (red) fibers for (a) SF regime $\left(9.7 \mathrm{~mJ} / \mathrm{cm}^{2}\right.$ with $0.5 \mathrm{~mm}$ spot size) and for (c) ASE regime (fluence of $0.02 \mathrm{~mJ} / \mathrm{cm}^{2}$ and $3 \times 3 \mathrm{~mm}^{2}$ spot size), excited from single laser pulse and measured simultaneously. Normalized emission strength from the zeroth LL versus shot number in the (b) SF regime and the (d) ASE regime. The dotted triangle is the emission

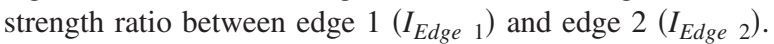

expected for ASE or SE. The correlation among two fiber output in Fig. 6(d) in clear contrast to Fig. 6(b) can be more visible in the case of dotted triangle as the emission strength

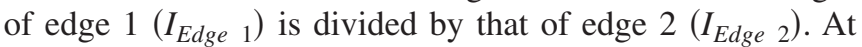
this lower power with a spot size much larger than the coherence length of SF (see Sec. IV C for the theoretical side), the 1-1 LL is barely seen while higher LLs are not occupied.

In order to study the importance of the coherence time of carriers in the SF formation process, we performed temperature-dependent measurements to vary the coherence time. In Fig. 7(a), we show emission spectra obtained at an elevated temperature of $110 \mathrm{~K}$ at various magnetic fields. As expected for SF, the threshold magnetic field increased significantly. Namely, we observe sharp features only above $\sim 23$ T. Figure 7(b) shows the temperature dependence of the threshold magnetic field $B_{T H}$ for the $0-0$ and 1-1 transitions. The solid lines are theoretical curves obtained through Eq. (9) taking into account dephasing due to phonon scattering, as described in Sec. V C.

Finally, we examined the characteristics of the narrow emission lines using broadened pulses. Inside a pulse com-
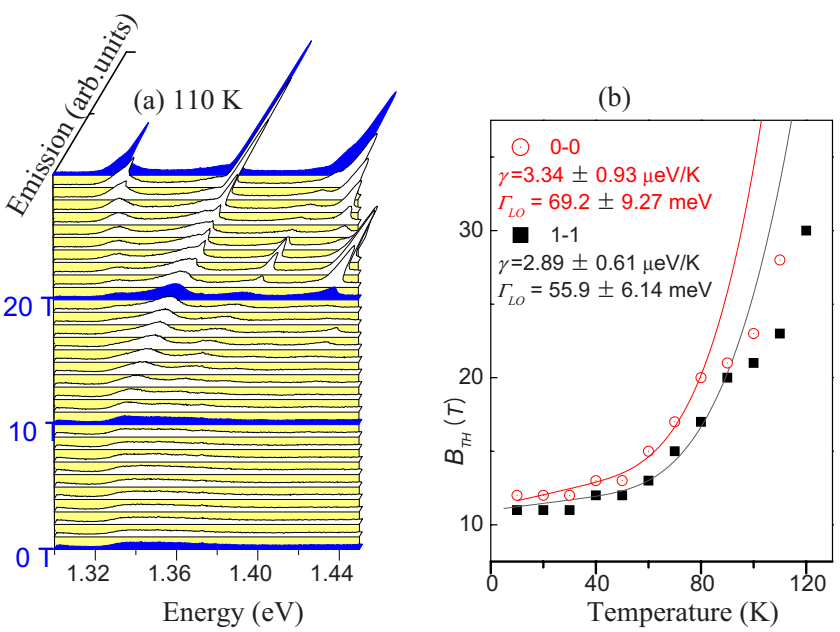

FIG. 7. (Color online) (a) Emission spectra at $110 \mathrm{~K}$ at various magnetic fields. (b) The threshold field $B_{\mathrm{TH}}(T)$ versus temperature ( $T$ ) for the $0-0$ LL (red circles) and for the 1-1 LL (black squares) emission features. The solid lines are fittings based on acoustic and optical phonon contributions from Eq. (9).

pressor of the regenerative amplifier system, the spacing between a grating pair was adjusted to increase the pulse width up to $30 \mathrm{ps}$; for the pulse compressor structure used within our laser system, see, e.g., Ref. 23. The sample temperature and laser fluence were fixed at $10 \mathrm{~K}$ and $\sim 0.1 \mathrm{~mJ} / \mathrm{cm}^{2}$, respectively. This value of fluence corresponds to a carrier density of $\sim 10^{13} \mathrm{~cm}^{-2}$. Figures 8 (a) shows corresponding emission spectra in the edge collection geometry with magnetic fields from 0 to $30 \mathrm{~T}$. The $1-1 \mathrm{LL}$ emission strength, compared with 0-0 LL, was much stronger than that of Fig. 1(b). This indicates that a certain amount of slow carrier relaxation from higher LL down to 0-0 LL exists in a temporal scale shorter than $30 \mathrm{ps}$. We also observe an oscillatory behavior at 1-1 and higher LLs, similar to that shown in Fig. 3 with 150 fs pulse width excitation. In Fig. 8(b), we traced the peak energy positions of the sharp Lorentzian feature for $0-0$ LL at different excitation pulse widths, which are seen to be redshifted from previously studied case of Gaussian-fitted CW absorption. ${ }^{19}$

\section{THEORY OF SUPERFLUORESCENCE IN MULTIPLE QUANTUM WELLS}

We analyze our measurements in the framework of SF in atomic two-level systems. The physics of SF in atomic systems has been studied for more than thirty years (see, e.g., Refs. 9, 10, and 24-26 and references therein). An incoherently prepared ensemble of excited two-level dipoles interacts via the exchange of spontaneously emitted photons. At sufficiently high densities $N$ of inverted atoms, this exchange leads to an effective mutual phasing of the atomic dipole oscillators. As a result, a macroscopic polarization with an amplitude $\propto N$ spontaneously forms, arising from quantum fluctuations, with a characteristic formation time, or delay time, $t_{d}$. The resulting macroscopic polarization decays by emitting a coherent radiation pulse radiation with duration $t_{p}$ scaling as $1 / N$ (in the simplest case) so that the emitted 

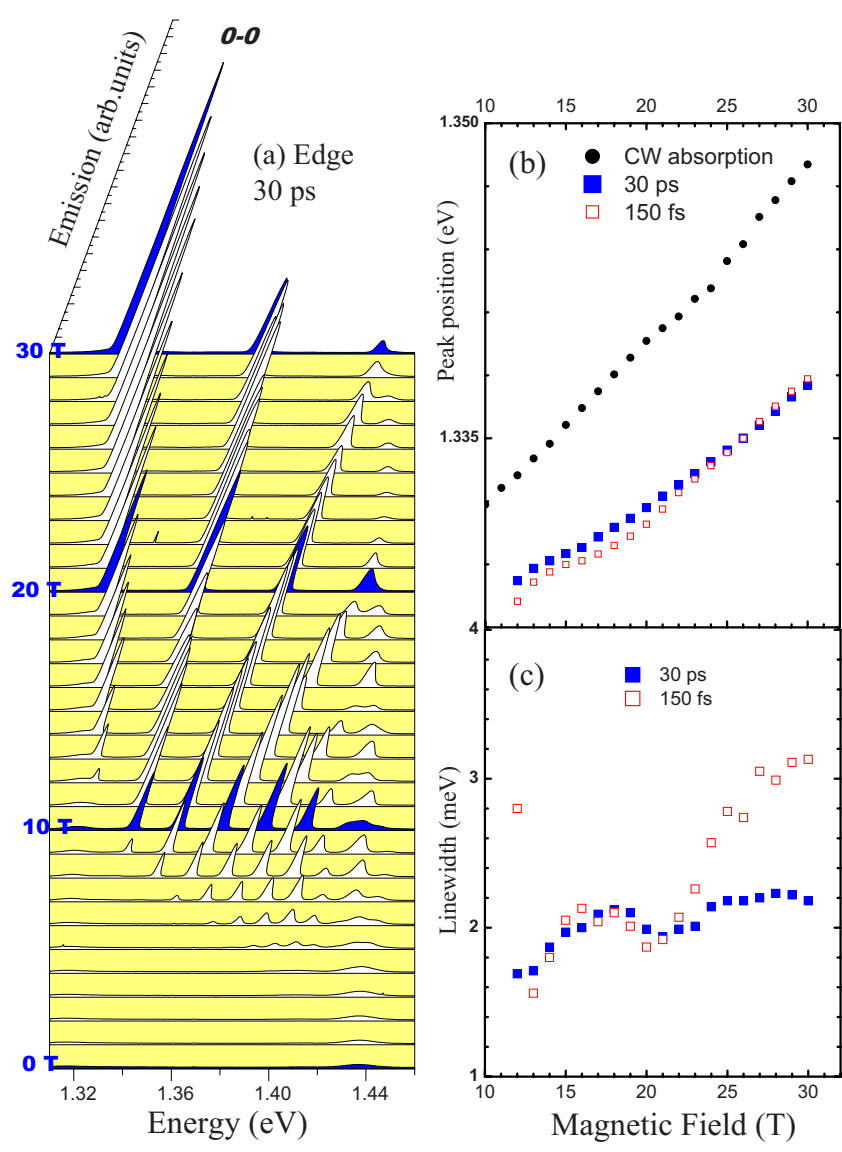

FIG. 8. (Color online) (a) Emission spectra at various of magnetic fields for an elongated excitation pulse width of $30 \mathrm{ps}$. (b) $0-0$ LL peak energy positions for pulse widths of $30 \mathrm{ps}$ (solid square) and 150 fs (open square), compared with low-power CW absorption. (c) Linewidth of the sharp feature from 0-0 LL versus $B$ for 30 ps (solid square) and $150 \mathrm{fs}$ (open square).

intensity $I_{\mathrm{SF}} \sim N / t_{p} \sim N^{2}$ exceeds by many orders of magnitude the intensity $I_{\mathrm{SE}}$ of spontaneous emission from the same number of incoherent atoms $\left(I_{\mathrm{SE}} \sim N / T_{1} \sim N\right)$.

The SF pulse duration $t_{p}$ and the delay time $t_{d}$ are shorter than the times of incoherent spontaneous emission and homogeneous phase relaxation in the medium: $t_{p}, t_{d}<T_{1}, T_{2}$. This is a distinctive feature of the phenomenon. In addition, SF is fundamentally a stochastic process: the optical polarization and the electromagnetic field grow from initially incoherent quantum noise to a macroscopic level. Thus, SF is intrinsically random: for identical preparation conditions, initial microscopic fluctuations get exponentially amplified and may result in the macroscopic pulse-to-pulse fluctuations in the delay time $t_{d}$, electric field polarization, and the direction of the emitted pulse.

We emphasize the difference between the two types of cooperative emission processes, superradiance and superfluorescence. Superradiance results when the formation of coherent polarization is driven by an external coherent laser field, whereas a superfluorescent macroscopic polarization develops spontaneously due to an intrinsic instability in a system of initially incoherent quantum dipole oscillators. Superradiance is generally easier to observe and has been previously observed in semiconductors. ${ }^{27}$
The key to achieving SF is to provide a high enough spatial and spectral density of inverted dipole oscillators (atoms, excitons, electron-hole pairs, etc.) such that the growth rate of the polarization exceeds the dephasing rate $\left(1 / T_{2}\right)$. In semiconductors, the rate of the optical polarization decay is typically $1-10 \mathrm{ps}^{-1}$ or greater, whereas the maximum density of electron-hole pairs within the bandwidth $\Delta \omega$ of the SF pulse is limited by the density of states $\rho(E): N_{\max }$ $\sim \rho(E) \hbar \Delta \omega$. It is this combination of a limited gain and fast decoherence that makes it difficult to achieve the threshold for SF. ${ }^{28,29}$ One possible way to overcome these limitations is to use quantum wells placed in strong magnetic fields. The combination of reduced dimensionality and the magnetic field fully quantizes the semiconductor into an atomiclike system with a series of LLs, thus strongly increasing the density of states. ${ }^{18}$ In addition, a complete quantum confinement and filling of all available electron states at the lowest LLs are expected to suppress the dephasing rate of the optical polarization. In our experiments, the initial pumping conditions are chosen so as to ensure the growth of coherence from an incoherent state by exciting the initial electron-hole plasma high in the bands with an excess energy of $150 \mathrm{meV}$ above the GaAs barrier band gap. The energy difference between the excitation energy and the $0-0 \mathrm{LL}$ in our $\mathrm{QWs}^{30}$ is greater than $270 \mathrm{meV}$, requiring many scattering events to relax into the QW LLs. The resulting electron-hole system is thus initially completely incoherent.

\section{A. Semiclassical model of superfluorescence}

To understand the threshold behavior and main features of $\mathrm{SF}$, we use a coupled density matrix and Maxwell equations in the semiclassical approximation, i.e., assuming a classical electromagnetic field. ${ }^{31}$ Note that although the optical polarization of an ensemble of quantum oscillators as a whole emerges spontaneously, individual atoms interact with a common electromagnetic mode through stimulated emission and absorption processes. As the field increases exponentially, a semiclassical treatment becomes applicable early in the process and determines its main features. This approach captures the main features of both exponential amplification and subsequent SF pulse formation in various systems. ${ }^{9}$

In our experiment, electrons and holes are created by a pump pulse high in the bands and then quickly lose energy, ending up in the ground subband of QWs before SF starts to develop. We do not consider the initial kinetics of rapid energy and momentum relaxation of carriers with a time scale of 10-500 fs since these hot carriers do not contribute to SF. We concentrate on carriers that have already reached a quasiequilibrium degenerate distribution over a few lowest LLs in the lowest subband. We furthermore consider radiation and optical polarization resulting from stimulated optical transitions between electrons and heavy holes on a given LL with occupation numbers $n_{i}$ and $p_{i}$.

Choosing $z$ as the quantum-well growth direction, we can write the electric field for $y$-polarized electromagnetic waves propagating in the $x$ direction as $E_{y}$ $=(1 / 2) E_{t}(y, z) A(x, t) \exp (i k x-i \omega t)$, where $E_{t}$ is a transverse distribution of the field. It satisfies a two-dimensional (2D) 
Helmholtz equation that determines transverse modes of the structure. For the slowly varying field amplitude $A$ of an electromagnetic mode near resonant to the given optical transition of frequency $\omega_{i}$ and the corresponding polarization amplitude $P$, one can obtain effective Maxwell-Bloch equations with rate equation terms for the incoherent decay of electron and hole populations

$$
\begin{gathered}
\frac{\partial A}{\partial x}+\frac{\mu}{c} \frac{\partial A}{\partial t}+\alpha A=\frac{2 \pi i \omega}{\mu c} \Gamma P, \\
\frac{\partial \rho_{0 i}}{\partial t}=-\left(\gamma_{i}+i \Delta_{i}\right) \rho_{0 i}+i \Omega_{R}\left(n_{i}+p_{i}-1\right), \\
\frac{\partial n_{i}}{\partial t}=\operatorname{Im}\left[\Omega_{R}^{*} \rho_{0 i}\right]+R_{e}-\frac{n_{i}}{T_{e}}-\frac{n_{i}-f_{e i}}{\tau_{e}}, \\
\frac{\partial p_{i}}{\partial t}=\operatorname{Im}\left[\Omega_{R}^{*} \rho_{0 i}\right]+R_{h}-\frac{p_{i}}{T_{h}}-\frac{p_{i}-f_{h i}}{\tau_{h}} .
\end{gathered}
$$

In Eq. (1), $\alpha=2 \pi \sigma /(\mu c)$ is the losses of a given electromagnetic mode with the background refractive index $\mu$, the nonresonant Ohmic conductivity of a sample at the optical frequency $\sigma(\omega)$, and the velocity of light in vacuum $c . \Gamma$ is an overlap of the transverse distribution of the electromagnetic field with the multiple quantum-well region. Here the amplitude of the polarization $P$ is related to the amplitude of the off-diagonal element of the density matrix $\rho_{i}=\rho_{0 i} e^{-i \omega t}$ as $P \simeq(1 / V) \sum_{i} d_{i} \rho_{0 i}$, where the summation is over all electronhole pairs with transition dipole moments $d_{i}$ on a given LL. Total volume densities $N_{e}$ and $N_{h}$ on a given LL are determined by a similar summation of $n_{i}$ and $p_{i}$. In Eq. (2), $\Delta_{i}$ $=\omega_{i}-\omega$ is the detuning of the frequency of the electromagnetic mode from the optical transition frequency $\omega_{i}$ and $\gamma_{i}$ is the transverse decay rate. $R_{e, h}$ are the rates of influx of optically excited carriers from upper states in the well and barriers and $T_{e, h}$ are total lifetimes of the spontaneous decay of a given state. The last terms in Eqs. (3) and (4) describe fast relaxation to quasiequilibrium distributions $f_{e, h}$ with time constants $\tau_{e, h}$, and $\Omega_{R}$ is the Rabi frequency of the electromagnetic mode. ${ }^{32}$

The Coulomb interaction renormalizes the optical transition frequencies and the Rabi frequency, and modifies all scattering terms; moreover it leads to additional off-diagonal terms as shown in the derivation of semiconductor Bloch equations. ${ }^{33,34}$ These effects are not essential for the magnitude of the SF growth rate and the SF dynamics which is mainly determined by resonant interaction of the field with electron-hole pairs on a given LL within a broad spectral width of a SF pulse.

The discrimination between $\mathrm{SF}$ and other radiation regimes is convenient to formulate in terms of a linear initialvalue problem, assuming constant initial degenerate populations of carriers created by pumping and subsequent relaxation. After finding the linear susceptibility $\chi(\omega)=P / A$ from Eq. (2) one can solve the dispersion relation following from Eq. (1) and find the complex frequency $\omega(k)$ as a function of a real wave number $k$. Note that the slow-varying amplitude approximation implies that $|\omega|$ and $k$ are close to the central resonance frequency $\omega_{0}$ of the LL transition and wave number $\mu \omega_{0} / c$, respectively. The instability, or exponential growth of the field and polarization with time, develops within the spectral interval of wave numbers where $\operatorname{Im}[\omega(k)]>0$. Obviously, for instability of any kind one needs positive population inversion with respect to stimulated recombination vs absorption processes at the photon frequency.

Consider first an idealized situation when all scattering rates and transition frequencies are the same for all electronhole pairs on a given LL, similarly to a homogeneously broadened active medium. Note that each spin-split LL has a degeneracy of $N_{2 \mathrm{D}}=e B /(2 \pi \hbar c) \simeq 2.4 \times 10^{10} B(T) \mathrm{cm}^{-2}$, and population inversion $\Delta N>0$ can be defined as the difference between occupied and unoccupied states per unit area. In this case, the dispersion relation $k \simeq \omega \mu / c+i \alpha+2 \pi \omega \chi(\omega) /(\mu c)$ which follows from Eq. (1) has two solutions $\omega(k)$ - eigenmodes that are sometimes called electromagnetic and polariton branches. ${ }^{9}$ Here $\chi(\omega) \approx i d^{2} \Delta N /\{\hbar[\gamma$ $\left.\left.+i\left(\omega_{0}-\omega\right)\right]\right\}$.

\section{B. Emission regimes and growth rates}

The key parameter governing the instability of these modes and different regimes of recombination is the cooperative frequency $\omega_{c}$ that determines the coupling strength between the field and the optical polarization as introduced in previous studies of SF in semiconductors ${ }^{29,35,36}$

$$
\omega_{c}=\sqrt{\frac{16 \pi^{2} d^{2} \Delta N \Gamma c}{\hbar \mu^{2} \lambda L_{\mathrm{QW}}}} .
$$

Here $L_{\mathrm{QW}}$ is the total width of the quantum wells (i.e., the sum of widths of all wells in the sample) and $\lambda$ is the wavelength of the radiated field. When the inversion $\Delta N$ is small or negative, field oscillations decay with time, and one can have only spontaneous recombination emission with power $\propto N_{e} / t_{s p}$, and the characteristic time scale $t_{s p} \sim 1 \mathrm{~ns}$, where $N_{e}$ at the lowest LL is close to the maximum (degeneracy) value in our experimental conditions. The inversion can be increased by increasing the fluence of the pumping pulse and the magnetic field strength. With increasing inversion and optical confinement, the modal gain may become higher than losses, and oscillations of the field and the polarization will grow with time.

There are two basic regimes of the instability, depending on the ratio between the values of $\omega_{c}$ and incoherent relaxation times. They correspond to the instability of one of the two eigenmodes existing in the medium. For low inversion density and fast decoherence, when $\omega_{c} \ll \gamma=1 / T_{2}$, one can have ASE, provided that photon losses $\alpha$ are low enough. This is a regime of a one-pass amplifier, corresponding to the instability of the electromagnetic branch. Its growth rate

$$
\operatorname{Im}(\omega)=g_{\mathrm{ASE}} \approx \frac{\omega_{c}^{2} \gamma}{4\left\{\gamma^{2}+(c / \mu)^{2}\left(k-k_{0}\right)^{2}\right\}}-c \alpha / \mu \ll \gamma
$$

is much slower than the dephasing rate $\gamma$. Equation (6) is obtained by substituting $\omega \approx c k / \mu$ in the expression for $\chi(\omega)$. This approximation is equivalent to calculating the net rate 
of stimulated transitions using the Fermi's golden rule.

When the gain increases to the value such that $g_{\text {ASE }} L \mu / c \gg 1$, amplification proceeds in the saturated regime, which is sometimes called superluminescence (SL). Here, $L$ is the length of an active medium in the propagation direction. The duration of the SL pulse in a saturated amplifier is $t_{\mathrm{SL}} \sim L \mu / c .^{26}$

If there is some feedback for any kind of modes existing in a sample, e.g., Fabry-Perot cavity modes formed by reflections from the edges or whispering gallery modes, then the system can lase on these modes. The growth rate of lasing modes will be defined by the same expression [Eq. (6)] with the optical confinement $\Gamma$ and photon lifetime $T_{E}$ defined for a given laser mode. One-pass amplification and lasing compete with each other, and the process with a higher growth rate dominates. We argue below that there is no feedback and therefore no lasing for our samples but it is important to stress here that all time scales for all of the above processes of ASE, SL, or lasing are longer than the dephasing time $T_{2}$. Under such conditions, the polariton mode is strongly damped with the decay rate $\gamma$, and one can adiabatically eliminate optical polarization from Eqs. (1)-(4) assuming $\partial \rho_{0} / \partial t=0$ and $|\Delta| \ll \gamma$. The polarization has a small amplitude and adiabatically follows the time dependence of the electromagnetic field. This implies low mutual coherence of dipole oscillations of individual electron-hole pairs.

Cooperative recombination or SF develops in the opposite limit of instability of the polariton branch. The growth rate of the polarization has to be larger than the decay rate $\gamma$. The instability results in the spontaneous formation of a largeamplitude coherent macroscopic polarization from initially incoherent oscillations of individual electron-hole dipole moments. Here "spontaneous" means that the polarization is not created by some external coherent laser field. The necessary condition for the instability is $\omega_{c}>2 \gamma$, or $\omega_{c}>2\left(\gamma \gamma^{*}\right)^{1 / 2}$, where the latter inequality is required for a system with a large inhomogeneous broadening $\gamma^{*} \gg \gamma$ of frequencies $\omega_{0}$ of electron-hole dipole oscillators. $9,29,35$

When the field decay rate is very high such that $\gamma$ $<\omega_{c} / 2<1 / T_{E}$, cooperative emission develops with a growth rate $g_{\mathrm{SF}} \approx \omega_{c}^{2} T_{E} / 4 \propto \Delta N$. Here $T_{E}$ is a total field decay time including both absorption in the medium and escape of radiation out of the sample. In this case the instability of the polariton branch occurs because of Ohmic dissipation (the dissipative instability). However, this case is not relevant for our samples that are characterized by a low field dissipation rate: $\omega_{c} / 2>\gamma>1 / T_{E}$. In the latter case, the growth rate saturates at its maximum value $g_{\mathrm{SF}} \approx \omega_{c} / 2 \propto(\Delta N)^{1 / 2}$. Note that in both cases the SF growth rate is faster than the phaserelaxation rate $\gamma$. This ensures maximally coherent nature of the process. In a sense, SF establishes an absolute upper limit on the rate with which an ensemble of initially incoherent inverted oscillators can radiate their stored energy.

The dynamics of SF are noteworthy and very different from ASE or lasing. First, SF develops within a broad spectral bandwidth $\sim g_{\mathrm{SF}}>\gamma$ and thus cannot be described by usual rate equations based on adiabatic elimination of the polarization. Second, after the degenerate (inverted) population of carriers is established on a given LL, the SF pulse is emitted with the delay time $t_{d} \sim\left(1 / g_{\mathrm{SF}}\right) \ln \left(I_{\mathrm{SF}} / I_{0}\right)$, which is logarithmically larger than the inverse growth rate, where the logarithm factor of order $10-20$ is due to the exponential growth of the field from the quantum noise level $I_{0}$ to the peak intensity $I_{\mathrm{SF}}$. This result can be obtained by solving Eqs. (1)-(4) neglecting all incoherent realization terms. Third, the pulse duration $\tau_{\mathrm{SF}} \sim 2 / g_{\mathrm{SF}}$ decreases with $\Delta N$, and therefore, the pulse intensity $I_{\mathrm{SF}} \sim N_{e} / \tau_{\mathrm{SF}}$ scales superlinearly $\propto N_{e}^{3 / 2} \propto B^{3 / 2}$ with electron density or the magnetic field, assuming that $\Delta N \sim N_{e}$. The coherence length over which an individual pulse is formed is given by $L_{c} \sim c t_{d} / \mu$, which is a logarithmic factor of order ten longer than the exponential amplification length $\sim c /\left(\mu g_{\mathrm{SF}}\right)$. Thus, the volume of the active medium in which all electron-hole dipoles become phased and contribute coherently into an exponential amplification of a given electromagnetic mode is given by $\sim \Gamma \lambda^{2} c /\left(\mu g_{\mathrm{SF}}\right)$. If we multiply this by the volume density of inverted oscillators $\Delta N / L_{\mathrm{QW}}$ and by the spontaneous recombination rate of an individual electron-hole pair given by $1 / t_{s p}=32 \pi^{3} d^{2} \mu /\left(\hbar \lambda^{3}\right)$, we obtain the maximum SF growth rate $\omega_{c} / 2$ within a factor of 2 .

The numerical value of the growth rate depends crucially on the optical confinement factor $\Gamma$. The contrast in the background refractive index $\Delta \mu \simeq 0.02$ between the MQW layer and GaAs is too low to provide wave guiding in a highly asymmetric waveguide formed by air on one side and GaAs on the other side of the MQWs. In the absence of a gaininduced change in the refractive index, the modes are spread over the whole thickness of the sample and the overlap factor $\Gamma$ is only $\sim 10^{-4}$. This small value of $\Gamma$ rules out all the possibility of SF. Numerical simulations show that the minimum contrast needed for wave guiding is $\Delta \mu=0.045$. The confinement factor $\Gamma$ is $\sim 0.1$ when $\Delta \mu=0.05$ and reaches 0.4 with subsequent increase in the index contrast to 0.09 when $B=25 \mathrm{~T}$. This affects the dependence of the ASE or SF growth rate and of the peak pulse power on the electron density or the magnetic field. Obviously, the dependence becomes stronger, which can be directly observed in timeresolved measurements.

\section{Gain guiding}

In an inverted medium, the polariton contribution to the background refractive index is positive on the blue side of the transition frequency $\omega_{0}$, with the peak index change $\delta \mu$ $\sim \pi d^{2} \Delta N /\left(\hbar \mu L_{\mathrm{QW}} \gamma\right)$ reached at a detuning $\omega-\omega_{0} \sim \gamma$. For a high-pumping fluence, we can assume that all states are occupied and $\Delta N=2 N_{2 \mathrm{D}}$; then for $T_{2}=1 / \gamma=250 \mathrm{fs}, \delta \mu$ reaches the wave guiding threshold of 0.03 when $B \simeq 12 \mathrm{~T}$. The latter value corresponds to the experimentally observed appearance of the narrow stimulated emission peak on the blue side of the broad spontaneous emission spectrum; see Fig. 1.

Thus, the polariton-supported wave guiding provides a natural explanation of the observed blueshifted peak. If we adopt this explanation, then the observed stimulated emission threshold can be used to determine $\gamma$. The number we obtained supports the suggestion that the dephasing time for quantum-confined degenerate carriers on the LLs before the formation of the emission pulse can be as long as several hundreds fs. Unfortunately, this value of $\gamma$ is difficult to 
verify independently, e.g., from the width of the spontaneous emission spectrum because $\gamma$ may change significantly during one emission cycle due to the varying density of carriers and we collected only time-integrated data. Also, there could be some inhomogeneous broadening of the spontaneous emission due to sample inhomogeneity within the pumped spot and the dependence of $\gamma$ on the carrier density. However, the width of the narrow stimulated emission peak does provide additional information on the values of $\gamma$ and the gain, which is consistent with the above estimate, as discussed below.

At the onset of wave guiding, stimulated recombination is still in the ASE regime, as discussed in more detail in Sec. IV A. The transition to SF occurs at $B \geq 20 \mathrm{~T}$, when the net growth rate $\omega_{c} / 2-\gamma \simeq 8 \times 10^{12} \mathrm{~s}^{-1}$ and $2 N_{2 \mathrm{D}} \simeq 10^{12} \mathrm{~cm}^{-2}$. The coherence length over which a SF pulse is formed is $L_{c}=c t_{d} / \mu \sim c /\left(\mu g_{\mathrm{SF}}\right) \ln \left(I_{\mathrm{SF}} / I_{0}\right)$. The ratio $I_{\mathrm{SF}} / I_{0}$ is on the order of the total number $N_{t}$ of electron-hole pairs contributing to a given pulse in the volume occupied by an electromagnetic mode, which we estimate as $N_{t} \sim 10^{7}$. Assuming $g_{\mathrm{SF}}$ $\sim 4 \times 10^{12} \mathrm{~s}^{-1}$, we obtain $L_{c} \sim 0.4 \mathrm{~mm}$. For observations of $\mathrm{SF}$, it is optimal to create an inverted (pumped) spot of this size. At much shorter lengths, a SF pulse does not have enough time/distance to develop, while at longer distances $L \gg L_{c}$ independent SF pulses will be formed at segments of length $L_{c}$, creating a noisy output over the time interval $\sim L \mu / c$. Note that in a sample of the optimal pumped length $L_{c}$ the shortest ASE pulse in a high gain regime has duration $\sim L_{c} \mu / c \sim 5$ ps, whereas a SF pulse is $\leq 1$ ps.

The observed spectral evolution of emission spectra as a function of electron-hole density, from a broad spontaneous emission to a narrow peak on the blue side of the SE line with subsequent further narrowing of the peak followed by broadening, is consistent with the above scenario. However, the observed spontaneous emission line has a full width of $\sim 9 \mathrm{meV}$, larger than the homogeneous linewidth of $5 \mathrm{meV}$ implied in the above discussion. The broad line may be caused by a variety of reasons, including the time evolution of the emission spectrum due to a changing density of carriers or spread of transition frequencies of electron-hole pairs. Assuming the latter, for an inhomogeneously broadened line, we numerically calculated the growth rate and gain-induced index change in electromagnetic waves for a Gaussian profile of inhomogeneous broadening with full width $2 \gamma^{*}$. Simulations show that the index change of 0.03 corresponding to the onset of wave guiding for the same magnetic field of 12 $\mathrm{T}$ is achieved when $2 \gamma^{*} \simeq 6 \mathrm{meV}$ and $2 \gamma \simeq 3 \mathrm{meV}$, i.e., it requires longer $T_{2}$. In this case the total $\mathrm{SE}$ linewidth is $\simeq 9 \mathrm{meV}$ and the maximum index is reached at the blueshift $\simeq 5 \mathrm{meV}$ from the central frequency.

After wave guiding is reached, the process enters a saturated SL regime accompanied by a line narrowing as a result of high-gain amplification. A diminishing linewidth is expected as the spectral components closest to the maximum of the gain spectrum get amplified stronger than components with greater detuning. An observed FWHM of the narrow peak changes with increasing electron-hole density from $2 \gamma \simeq 5 \mathrm{meV}$ to $2 \Delta \omega \simeq 2 \mathrm{meV}$. If the maximum gain [Eq. (6)] at the center of the gain spectrum is equal to $g_{0}$, it is easy to obtain the linewidth

$$
\frac{\Delta \omega}{\gamma}=\left\{\frac{g_{0} \mu / c}{\ln \left[\left(G_{0}+1\right) / 2\right]}-1\right\}^{1 / 2},
$$

where $G_{0}=\exp \left[g_{0} L \mu / c\right]$. For $L=0.5 \mathrm{~mm}$ (the pump spot size), the observed narrowing by a factor of $\sim 2.5$ is consistent with the observed net amplification by a factor of order 100 .

The transition to SF is accompanied by shortening of the pulse from $\tau_{\mathrm{ASE}} \sim L \mu / c \approx 5$ ps to $\tau_{\mathrm{SF}} \sim 4 / \omega_{c} \leq 2 / \sqrt{\gamma \gamma^{*}}$ $\leq 1$ ps. This shortening of the pulse duration leads to the observed line broadening when the pulse duration becomes smaller than the inverse total bandwidth of the stimulated emission peak. If we identify $2 \hbar / \tau_{\mathrm{SF}} \sim \hbar g_{\mathrm{SF}}$ with the observed emission full width of $2.5 \mathrm{meV}$ at $B=25 \mathrm{~T}$ in Fig. 2(b), the value of the cooperative frequency is in the range 7-9 meV, depending on the type of broadening we assume. This value is definitely higher than both $\hbar \gamma$ and $\hbar \sqrt{\gamma \gamma^{*}}$. Thus, the observed spectral behavior is highly suggestive of a continuous evolution from a regime where ASE is the primary emission mechanism to an SF-dominated regime.

\section{DISCUSSION}

\section{A. Superlinear scaling with magnetic field and fluence:} Evidence for multiple superfluorescence-pulse formation

The intensity of a single SF pulse from a single LL is expected to scale with the electron density $N_{e}$ or the magnetic field $B$ as $I_{\mathrm{SF}} \sim \hbar \omega \Delta N / \tau_{\mathrm{SF}} \propto N_{e}^{3 / 2}\left[\Gamma\left(N_{e}\right)\right]^{1 / 2}$ or $B^{3 / 2}[\Gamma(B)]^{2}$, respectively, where $\Gamma$ is the overlap factor as introduced in Eq. (1), if it is assumed that the pump pulse fluence $J_{p} \propto \Delta N \propto N_{e}$. However, we cannot directly probe this superlinear scaling since the emission strength is integrated over $1 \mathrm{~s}$ (1000 shots). Assuming all emitted photons are collected, we should observe a linear dependence of the integrated emission strength from $N_{e}$ or $B$, which contradicts the observed superlinear dependence in the SF regime. See Fig. 2.

We interpret the superlinear scaling of time-integrated intensity of SF from 0-0 Landau level as the development of multiple SF pulses after each pumping shot. As the first SF pulse develops on the 0-0 LL and leads to a fast depletion of electron-hole pairs on this level on a subpicosecond scale, electron-hole pairs on the 1-1 LL do not have time to recombine via a much slower ASE process. Thus, they lose energy by relaxing to the zeroth level and emit another SF pulse, leading to superlinear increase in the total fluence from the zeroth level.

There are several additional points that support this proposed scenario of multiple SF pulse formation. First, the superlinear scaling is observed only for the case of $0.5 \mathrm{~mm}$ spot size (see Fig. 4), approximately the SF coherence length $L_{c}$ as discussed in more detail above. SF cannot develop for spot sizes smaller than the coherence length. On the other hand, for larger spot sizes, many independent, uncorrelated SF pulses are created, with the total integrated emission resembling ASE rather than SF. Furthermore, the superlinear increase in the $0-0$ LL emission is accompanied by a decrease in emission from higher LLs below $0.25 \mathrm{~mJ} / \mathrm{cm}^{2}$. Finally, the statistics of counts received by two edges in Fig. 
6 is consistent with the formation of two pulses from the $0-0$ LL. At very high pump fluences, SF may develop from two or three LLs simultaneously and the growth of the emission strength with the pump fluence and magnetic field should slow down.

In Fig. 4(b), we observe a scaling behavior similar to the field scaling of $0-0$ LL shown in Fig. 2(b) - the integrated strength (black squares) evolves from a threshold of $0.01 \mathrm{~mJ} / \mathrm{cm}^{2}$ through a linear regime (green line; $0.01-0.03 \mathrm{~mJ} / \mathrm{cm}^{2}$ ) to a superlinear regime (blue line; $0.03-0.2 \mathrm{~mJ} / \mathrm{cm}^{2}$ ). Above $0.2 \mathrm{~mJ} / \mathrm{cm}^{2}$ in Fig. 3(b), the signal resumes a linear scaling after the SF from 0-0 LL is saturated as the level is fully filled. The sustained linear regime in the $0-0$ LL is possibly associated with additional carriers passed on from higher LL in the later stage after the SF pulse burst. The carrier density which produces the linear scaling regime after saturation can only be sufficient for ASE but not for SF. Such carrier depletion from higher to lower LL was also manifested in Fig. 2(a) via anticorrelation between $0-0$ and $1-1 \mathrm{LL}$ above $20 \mathrm{~T}$.

\section{B. Stochastic nature of superfluorescent emission}

The strongest evidence for SF at high-pumping fluences and magnetic fields in our samples comes from the random directionality experiment in the saturated SF regime ( $>0.2 \mathrm{~mJ} / \mathrm{cm}^{2}$ ); see Fig. 6. In this experiment, the pumped spot size was equal to one coherence length $(0.5 \mathrm{~mm})$ and radiation was collected after single pulse excitation from two perpendicular edges simultaneously. Since the pumped area is a circle, both SE and ASE should be emitted in all directions with the same intensity and without any correlation or anticorrelation effects. However, this is no longer true for SF. In the development of SF, initial quantum fluctuations grow to a macroscopic level and lead to strong fluctuations of the delay time from pulse to pulse. Initially, all propagation directions are equivalent. However, after one pulse has propagated in one direction, all electron-hole pairs are consumed along its path. Therefore, formation of the second pulse in the direction traversing the path of the first pulse becomes suppressed. Indeed, a SF pulse consumes virtually all electron-hole pairs along its path leaving a narrow (of a few $\mu \mathrm{m}$ width) unpumped stripe with negative inversion $\Delta N$. This stripe is antiguiding for the blueshifted SF emission. This means that the SF radiation from any delayed pulse crossing the depleted stripe will spread over the whole thickness of the sample after just one wavelength $\lambda / \mu$ $\sim 0.3 \mu \mathrm{m}$ of propagation length. This reduces the overlap with an MQW layer to $\Gamma \sim 10^{-4}$, thus effectively quenching the pulse. Therefore, we should observe strong anticorrelation between the emission strengths received by the two edges after each pumping shot.

The data can be best explained if there are two consecutive SF pulses that can be formed per each pump shot, according to the scenario suggested above. Each pulse can go either to edge 1 or to edge 2. Evidently, there are also pulses generated in the opposite direction that we do not detect. At the same time, our collecting prisms were very wide (1 $\times 1 \mathrm{~mm}^{2}$ ) so we collected most of the radiation propagating toward each of the two edges. Then, in 50\% events, both edges received an SF pulse, or at least some radiation if there is not enough electrons for the second SF pulse, and this second pulse is actually ASE. In the other 50\% events, only one edge will receive both pulses.

\section{Temperature dependence}

Because the formation of SF inherently relies on the establishment of a macroscopic coherence among the photoexcited carrier density, we expect that the emission threshold and strength should depend sensitively on the temperature. An increasing phonon number with temperature should increase the dephasing rate $T_{2}$ and thus increase the threshold magnetic field and carrier density, as the experimental data in Fig. 7 suggest.

As we follow the threshold field $B_{\mathrm{TH}}(T)$ for $0-0 \mathrm{LL}$ (red circles) and for 1-1 LL (black squares) sharp feature in Fig. $7(\mathrm{~b})$, we observe that it is more rapidly increasing above 50 $\mathrm{K}$. Here, we define $B_{\mathrm{TH}}$ as the critical field for the appearance of narrow emission lines. Even though the $B_{\mathrm{SF}}$ is not coincident with $B_{\mathrm{TH}}$ since the sharp emission feature is generated by both the ASE and SF, we will presume that their temperature variation should be similar. First, note that the critical magnetic field $B_{\mathrm{SF}}$ for SF generation is obtained from the relation between $\omega_{c} / 2(\propto \sqrt{N})$ and the dephasing rate $1 / T_{2},{ }^{18,35} \omega_{c} / 2>1 / T_{2}$. The LLs density of states is given by $N=e B / h$, i.e., proportional to $B$ and therefore to $\omega_{c}^{2}$. For electron-phonon scattering, the dephasing rate $1 / T_{2}$ can be expressed phenomenologically as

$$
1 / T_{2} \propto \Gamma_{0}+\gamma T+\Gamma_{\mathrm{LO}} /\left[\exp \left(E_{\mathrm{LO}} / k_{B} T\right)-1\right],
$$

where $\Gamma_{0}$ is the width due to the inhomogeneous broadening and $\gamma\left(\Gamma_{\mathrm{LO}}\right)$ is a parameter that measures the interaction with acoustic phonons (polar LO phonons). Thus, writing $e B_{\mathrm{SF}} / h \propto \omega_{c}^{2}=\left(2 / T_{2}\right)^{2}$, we find

$$
B_{\mathrm{SF}} \propto\left(\Gamma_{0}+\gamma T+\Gamma_{\mathrm{LO}} /\left[\exp \left(E_{\mathrm{LO}} / k_{B} T\right)-1\right]\right)^{2} .
$$

Since the LO phonon energy $E_{\mathrm{LO}}$ in our sample structure, which is expected to be similar to that of GaAs-based QW $(\sim 36 \mathrm{meV})$, is much larger than the thermal energy $\left(k_{B} T\right)$ in our temperature range, we tentatively identify the acoustic phonon contribution as the dominant temperature mechanism for varying $B_{\mathrm{SF}}$. The red (black) curve in Fig. 7(b) is the fitting based on Eq. (9) for 0-0 LL (1-1 LL), where we can obtain the comparative values of $\gamma / \Gamma_{0}$ and $\Gamma_{\mathrm{LO}} / \Gamma_{0}$, respectively. When $\Gamma_{0}$ is assumed to be the same as the minimum linewidth obtained from Fig. 2(b) $(=1.9 \mathrm{meV}), \gamma\left(\Gamma_{\mathrm{LO}}\right)$ is smaller (larger) than that of the two-dimensional exciton case by two to three times (three to four times), ${ }^{37}$ while very similar to the zero-dimensional case. ${ }^{38}$ The deviation of the fitting curve above $80 \mathrm{~K}$ for $0-0 \mathrm{LL}$ and above $90 \mathrm{~K}$ for $1-1$ LL is possibly associated with carrier delocalization and/or deionized impurities at high temperatures. ${ }^{39} \Gamma_{0}(\sim 1.9 \mathrm{meV})$ indicates that the SF pulse duration is shorter than $700 \mathrm{fs}$ ( $\left.=2 \hbar / \Gamma_{0}\right)$ while the temperature dependence of $B_{\mathrm{TH}}(T)$ agrees with the dephasing dynamics in a zero-dimensional system. 


\section{Pump pulse-width dependence}

Finally, we investigated how the emission lines depend on the excitation pulse width, as compared with the delay time $t_{d}$. For the optimal coherence length $L_{c}, t_{d}$ is roughly estimated to be $\sim 10$ ps when the pumping fluence of $\sim 0.1 \mathrm{~mJ} / \mathrm{cm}^{2}$ almost saturates the available states in $0-0 \mathrm{LL}$ [cf. Fig. 4(b)]. ${ }^{36}$

The red-shifted peak position for pulsed excitation indicates not only the existence of localized states in these strained samples ${ }^{19}$ but also band-gap renormalization. ${ }^{40,41}$ We also note that the effect of band-gap renormalization was stronger at $150 \mathrm{fs}$ pulse but the difference between $150 \mathrm{fs}$ and 30 ps was almost suppressed above $25 \mathrm{~T}$. The $150 \mathrm{fs}$ pulse creates carriers more abruptly than 30 ps pulse and the following carrier-carrier interactions will be enhanced to screen the excitonic Coulomb interactions more effectively. The disappearance of the peak energy separation between $150 \mathrm{fs}$ and $30 \mathrm{ps}$ above $25 \mathrm{~T}$, therefore, could be possibly associated with combined effects of increased carrier density and reduced screening effects because of more strongly confined wave functions via $B$ increment. The slope of peak position versus $B$ in Fig. 8(b) became more similar to the $\mathrm{CW}$ absorption case with the increasing field, which suggests a reduction in effective-mass renormalization in spite of increased carrier density at higher fields. More details on the field-dependent variation in band gap and effective-mass renormalization will be discussed elsewhere.

To further characterize the emission lines in Fig. 8(c), we measured the linewidths for 150 fs and for 30 ps for Lorentzian sharp features in 0-0 LL. Figures 8(c) displays the clear trend of increasing linewidths with $B$ for $150 \mathrm{fs}$ in contrast to those for $30 \mathrm{ps}$. As discussed earlier, $30 \mathrm{ps}$ is much longer than the estimated delay time $t_{d}$ to build coherence within $L_{c}$ in our system and the intrarelaxation time on the order of 500 fs. ${ }^{40}$ Since the increment of linewidth with $B$ was a signature of SF, the observed plateau in the linewidth trace for $30 \mathrm{ps}$ pumping in Fig. 8(c) implies saturated amplified spontaneous emission, not reaching the SF regime yet.

\section{CONCLUSIONS}

We have performed magnetophotoluminescence measurements in $\operatorname{In}_{x} \mathrm{Ga}_{1-x} \mathrm{As} / \mathrm{GaAs}$ multiple quantum wells in high magnetic fields using intense femtosecond pulses. The resulting density and energy confinement is sufficient to generate a spontaneous macroscopic polarization that decays through the emission of SF pulses. Our experiments demonstrate multiple evidence for the cooperative SF regime of spontaneous recombination as revealed by the spectral behavior, magnetic field and fluence scalings, spot size dependence, and spatial and temporal correlations. We have further probed the appropriate conditions for observing SF by exploiting its spectral features and dependence on the temperature and focal geometry.

\section{ACKNOWLEDGMENTS}

This work was supported by the Korean Research Foundation under Grant No. (MOEHRD, KRF-2007-313C00218), the NSF ITR program (Grant No. DMR-032547) and the Bio-imaging Research Center at GIST. A portion of this work was performed at the National High Magnetic Field Laboratory, supported by NSF Cooperative under Agreement No. DMR-0084173 and by the State of Florida. A.B. acknowledges the support from the NSF CAREER program (Grant No. 0547019) and NSF PIRE (Grant No. OISE0530220) and ERC programs.

\footnotetext{
*jho@gist.ac.kr

${ }^{1}$ See, e.g., J. Shah, Ultrafast Spectroscopy of Semiconductors and Semiconductor Nanostructures (Springer-Verlag, Berlin, 1999).

${ }^{2}$ Y. Yamamoto and A. Imamoglu, Mesoscopic Quantum Optics (Wiley, New York, 1999).

${ }^{3}$ S. T. Cundiff, A. Knorr, J. Feldmann, S. W. Koch, E. O. Gobel, and H. Nickel, Phys. Rev. Lett. 73, 1178 (1994).

${ }^{4}$ T. Tokihiro, Y. Manabe, and E. Hanamura, Phys. Rev. B 51, 7655 (1995)

${ }^{5}$ D. S. Jin, J. R. Ensher, M. R. Matthews, C. E. Wieman, and E. A. Cornell, Phys. Rev. Lett. 77, 420 (1996).

${ }^{6}$ See, e.g., P. G. de Gennes, Superconductivity of Metals and Alloys (Benjamin, New York, 1966).

${ }^{7}$ M. Greiner, O. Mandel, T. Esslinger, T. W. Hansch, and I. Bloch, Nature (London) 415, 39 (2002).

${ }^{8}$ See, e.g., A. E. Siegman, Lasers (University Science Books, Sausalito, 1986), p. 549.

${ }^{9}$ V. V. Zheleznyakov, V. V. Kocharovsky, and Vl. V. Kocharovsky, Sov. Phys. Usp. 32, 835 (1989).

${ }^{10}$ R. Bonifacio and L. A. Lugiato, Phys. Rev. A 11, 1507 (1975).

${ }^{11}$ R. H. Dicke, Phys. Rev. 93, 99 (1954).
}

${ }^{12}$ N. E. Rehler and J. H. Eberly, Phys. Rev. A 3, 1735 (1971).

${ }^{13}$ N. Skribanowitz, I. P. Herman, J. C. MacGillivray, and M. S. Feld, Phys. Rev. Lett. 30, 309 (1973).

${ }^{14}$ H. M. Gibbs, Q. H. F. Vrehen, and H. M. J. Hikspoors, Phys. Rev. Lett. 39, 547 (1977).

${ }^{15}$ R. Florian, L. O. Schwan, and D. Schmid, Solid State Commun. 42, 55 (1982).

${ }^{16}$ P. V. Zinoviev, S. V. Lopina, Yu. V. Naboikin et al., Sov. Phys. JETP 58, 1129 (1983).

${ }^{17}$ M. S. Malcuit, J. J. Maki, D. J. Simkin, and R. W. Boyd, Phys. Rev. Lett. 59, 1189 (1987)

${ }^{18}$ Y. D. Jho, X. Wang, J. Kono, D. H. Reitze, X. Wei, A. A. Belyanin, V. V. Kocharovsky, Vl. V. Kocharovsky, and G. S. Solomon, Phys. Rev. Lett. 96, 237401 (2006).

${ }^{19}$ Y. D. Jho, F. V. Kyrychenko, J. Kono, X. Wei, S. A. Crooker, G. D. Sanders, D. H. Reitze, C. J. Stanton, and G. S. Solomon, Phys. Rev. B 72, 045340 (2005).

${ }^{20}$ G. Tränkle, H. Leier, A. Forchel, H. Haug, C. Ell, and G. Weimann, Phys. Rev. Lett. 58, 419 (1987).

${ }^{21}$ L. V. Butov, V. D. Kulakovskii, E. Lach, A. Forchel, and D. Grutzmacher, Phys. Rev. B 44, 10680 (1991). 
${ }^{22}$ M. Potemski, J. C. Maan, K. Ploog, and G. Weimann, Solid State Commun. 75, 185 (1990).

${ }^{23}$ B. Loiseaux, A. Delboulbé, J. P. Huignard, P. Tournois, G. Cheriaux, and F. Salin, Opt. Lett. 21, 806 (1996).

${ }^{24}$ M. Gross and S. Haroche, Phys. Rep. 93, 301 (1982).

${ }^{25}$ A. V. Andreev, V. I. Emelyanov, and Yu. A. Ilyinskii, Cooperative Effects in Optics (IOP, Bristol, 1993).

${ }^{26}$ M. G. Benedict, A. M. Ermolaev, V. A. Malyshev, I. V. Sokolov, and E. D. Trifonov, Superradiance. Multiatomic Coherent Emission (Institue of Physics, Bristol, 1996).

${ }^{27}$ See, e.g., D. Ammerlahn, J. Kuhl, B. Grote, S. W. Koch, G. Khitrova, and H. Gibbs, Phys. Rev. B 62, 7350 (2000).

${ }^{28}$ A. A. Belyanin, V. V. Kocharovsky, and Vl. V. Kocharovsky, Solid State Commun. 80, 243 (1991); Laser Phys. 2, 952 (1992).

${ }^{29}$ A. A. Belyanin, V. V. Kocharovsky, and Vl. V. Kocharovsky, Quantum Semiclassic. Opt. 9, 1 (1997).

${ }^{30}$ The high-field Landau notation is used to specify a QW electronhole plasma in a magnetic field; for low-field-high-field correspondence, see, e.g., A. H. MacDonald and D. S. Ritchie, Phys. Rev. B 33, 8336 (1986).

${ }^{31}$ Although the very initial stage of SF requires full quantum electrodynamic analysis, the semiclassical approximation becomes valid as soon as the number of photons per mode becomes larger than one.
${ }^{32} \Omega_{R}$ also contains nonresonant "local field" corrections from screened Coulomb interactions. However, we will neglect them here, assuming $\Omega_{R}=d A / 2 \hbar$, where the dipole moment $d / e$ $\simeq 0.5 \mathrm{~nm}$. The local field effect can be important for $\mathrm{SF}$ of a dense small sample of size much smaller than the wavelength. In our case it creates only a small correction to the SF dynamics.

${ }^{33} \mathrm{H}$. Haug and S. W. Koch, Quantum Theory of the Optical and Electronic Properties of Semiconductors (World Scientific, Singapore, 1994).

${ }^{34}$ M. W. Wu and H. Haug, Phys. Rev. B 58, 13060 (1998).

${ }^{35}$ A. A. Belyanin, V. V. Kocharovsky, and V1. V. Kocharovsky, Quantum Semiclassic. Opt. 10, L13 (1998).

${ }^{36}$ A. A. Belyanin et al., Laser Phys. 13, 161 (2003).

${ }^{37}$ H. Zhao, S. Wachter, and H. Kalt, Phys. Rev. B 66, 085337 (2002).

${ }^{38}$ I. Favero, G. Cassabois, R. Ferreira, D. Darson, C. Voisin, J. Tignon, C. Delalande, G. Bastard, Ph. Roussignol, and J. M. Gérard, Phys. Rev. B 68, 233301 (2003).

${ }^{39}$ J. Lee, E. S. Koteles, and M. O. Vassell, Phys. Rev. B 33, 5512 (1986).

${ }^{40}$ M. C. Tatham, J. F. Ryan, and C. T. Foxon, Phys. Rev. Lett. 63, 1637 (1989).

${ }^{41}$ L. V. Butov, V. D. Egorov, V. D. Kulakovskii, and T. G. Andersson, Phys. Rev. B 46, 15156 (1992). 